\title{
Comparative Study of Acoustic Beam forming Techniques for Removal of Interference
}

\author{
Devi Sivaraman \\ VLSI Group \\ Department of EECE \\ ITM University, Gurgaon \\ (Haryana) India
}

\author{
Neeraj Kr. Shukla \\ VLSI Group \\ Department of EECE \\ ITM University, Gurgaon \\ (Haryana) India
}

\author{
Ajith Kumar P.C \\ SBU Group TATA ELXSI \\ Thiruvananthapuram \\ (Kerala) India
}

\begin{abstract}
Beam forming system helps in quality communication by increasing the signal to noise ratio (SNR). The purpose of this system is to recover the source signal from the interference. This paper explains the concept and algorithms of beam forming based on review of relevant literature. Conventional methods as well as advanced algorithms regarding acoustic beam forming are discussed. There are basically two types of beam formers, signal independent beam formers or conventional beam formers and signal dependent beam formers or adaptive beam formers. The main purpose is to allow signal from a desired direction and to remove interference. This paper theoretically compares the features of various techniques, to enable the use of appropriate ones for acoustic beam forming.
\end{abstract}

\section{Keywords}

Beam forming, Delay and Sum, MVDR, MUSIC, Root MUSIC, ESPRIT, Least Mean Square.

\section{INTRODUCTION}

The beam forming system improves the quality of communication by increasing the signal to noise ratio (SNR). The system recovers the source signal from the interference. In acoustic systems the performance of distant-talking applications is difficult making it more susceptible to distortion from noise. De-reverberation and noise removal are essential for the improvement of signal quality. This could be done using a two stage beam former where the first stage concentrates on reducing reverberation while the second stage removes noise [1]. In these situations, microphone arrays are used to spatially filter the source signal from the noise [2]. The beam forming system can also utilize a Field Programmable Gate Array (FPGA) for high-throughput, real time and modular beam forming [3]. The concept behind this is constructive interference and destructive interference, the former reinforces the speech signal while the later is used to remove noise. There could be adaptive as well as nonadaptive beam formers [4]. A basic beam forming system is shown in Fig.1. A wide band constant beam width beam former is most desirable as its beam width remains constant for all frequency bands.

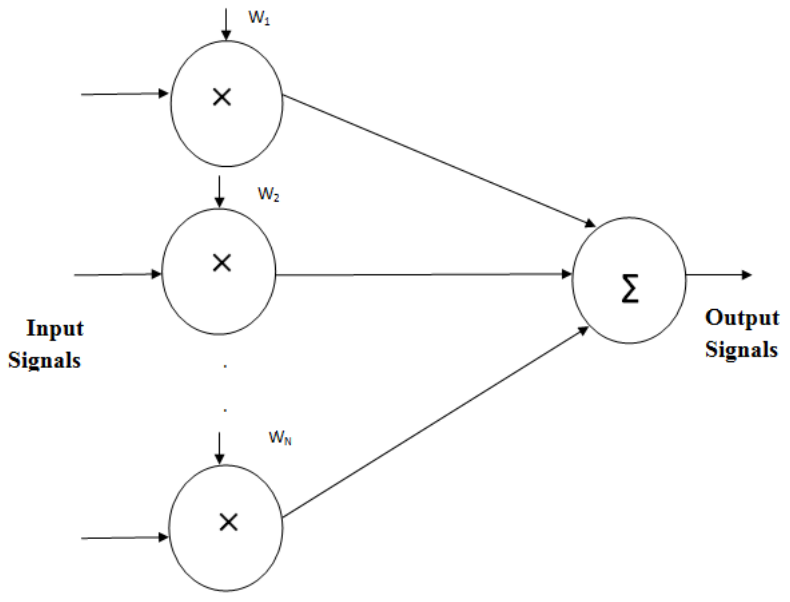

Fig.1 General Beam forming system

The purpose of this paper is to compare theoretically the different algorithms of acoustic beam forming.

\section{REVIEW OF BEAM FORMING METHODS}

This session deals with the different methods and algorithms of acoustic beam forming. There are different algorithms for performing beam forming which can be classified into conventional and advanced as explained below.

\subsection{Conventional Method}

The conventional form of beam forming technique can be better explained through a delay and sum beam former. The basic idea is to delay or advance the signal from microphone arrays by an amount of time so that synchronization across all sensors is obtained [4]. A uniform linear array is used for this method where the distance between source and array is much greater than the length of the array. However, the microphones are considered in such a way that the gain does not vary. The first microphone is considered as the reference and the distance between microphones are at a fixed distance of 'd' with a particular source incidence angle. Output from each microphone is a multiplied result of gain and signal with the appropriate delay where the delay is calculated with the help of 'd' and source angle incidence $\Theta$, according to Eq.1 [5].

Delay $=(\mathrm{N}-1) * d * \operatorname{Sin}(\Theta) /$ C. .....Eq. 1

Where Delay $=$ time delay experienced at each microphone by the input signal

$$
\begin{aligned}
& \mathrm{N}=\text { number of microphones in the array } \\
& \mathrm{C}=\text { velocity of sound in air } \\
& \mathrm{O}=\text { direction of arrival of source signal }
\end{aligned}
$$


The delay and sum beam former is basically used for narrow band signal. To perform on broad band signal, Fast Fourier Transform (FFT) is used. This (FFT) would help in decomposing the signal into narrow band on which beam forming is performed [5]. The basic principle is in maximizing the source signal power.

\subsection{Advanced Method}

Adaptive beam forming is a technique employed by a series of antenna arrays to select a desired signal from one direction while eliminating all other signals of the same frequency from other directions. In this technique, weight vectors are iteratively calculated based on some simple or complex algorithms using covariance or correlation matrix obtained from unknown statistics to get the unknown weight coefficient. The optimality criteria are to maximize signal to interference ratio [6]. The various adaptive methods used in acoustic beam forming include Minimum-variance Distortionless Response (MVDR), MUSIC Algorithm, Root-Music Algorithm, ESPRIT Algorithm, Least Mean Square (LMS) etc [5].

\section{i. MVDR Beam Forming}

MVDR Beam Forming minimizes the power of noise signals coming from directions other than source signal direction. This technique does not maximize power as that of conventional beam former but nullifies the power in directions other than the look direction and also keeps fixed gain in the look direction [5]. This beam former is based on Mean Square Error (MSE) criteria and rejects maximum level of noise allowable without distorting the desired signal at each frequency [1]. The weight calculation is given by

$\mathrm{W}=\left(\mathrm{R}^{-1 *} \mathrm{a}(\Theta)\right) /\left(\mathrm{a}^{\mathrm{H}}(\Theta) * \mathrm{R}^{-1} \mathrm{a}(\Theta)\right) \ldots \ldots \ldots . .$. Eq. 2 [6] [7] [8]

Where $\mathrm{W}=$ weight coefficient

$$
\begin{aligned}
& a=\text { steering vector } \\
& R=\text { correlation matrix } \\
& \Theta=\text { direction of arrival of source signal }
\end{aligned}
$$

In MVDR, the constraint for weight calculation ensures that the signal from a particular look direction passes undistorted, while interference signals are suppressed.

So the output signal power will be same as source power. The main disadvantage of this method is that it cannot be used for multipath signals as it sensitizes signals from a single direction only [7].

The steering vector and correlation matrix are important parameters in calculation of weight coefficients. Steering vector is given by

$\mathrm{a}(\Theta)=\left[1 \mathrm{e}^{\left(-\mathrm{j}^{*} 2^{*} \pi^{*} \mathrm{f}^{*} \mathrm{~d}^{*} \sin (\Theta) / \mathrm{c}\right)} \ldots . \mathrm{e}^{\left(-\mathrm{j} *(\mathrm{M}-1)^{*} 2^{*} \pi^{*} \mathrm{f}^{*} \mathrm{~d}^{*} \sin (\Theta) / \mathrm{c}\right)}\right] / \sqrt{ } \mathrm{M} \ldots$... Eq.3

Where $f=$ frequency of signal

$\mathrm{d}=$ distance between sensors

$\mathrm{M}=$ number of sensors

This help in steering the filter to the required beam direction [6].

Correlation matrix is calculated using input signal, $\mathrm{x}$.

$\mathrm{R}=(1 / \mathrm{M}) * \sum_{\mathrm{k}=1-\mathrm{M}} \mathrm{x}(\mathrm{k}) * \mathrm{x}^{\mathrm{H}}(\mathrm{k})$ .Eq.4 [6]

The MVDR is a special case of Linearly Constraint Minimum Variance Beam former (LCMV) where the constraint to obtain a signal in a particular direction [8] is given by

$$
\begin{aligned}
& \sum_{\mathrm{K}=0 \text { toM- } 1}\left(\mathrm{w}(\mathrm{K}) *\left(\mathrm{e}^{-\mathrm{j} \mathrm{k} \Omega}\right)\right)=\mathrm{w}{ }^{\mathrm{H}_{*}} \mathrm{a}(\Omega)=\mathrm{g} \ldots \ldots \ldots \ldots \ldots \text { Eq.5 [6] } \\
& \text { Where } \Omega=2 * \mathrm{pi}^{*} \mathrm{f}^{*} \mathrm{~d} * \sin (\Theta) / \mathrm{c} \\
& \quad \mathrm{g}=\text { gain }=1
\end{aligned}
$$

This gives the weight vector as given by Eq.2 while output signal is given by,

$$
\mathrm{y}(\mathrm{n})=\mathrm{s}(\mathrm{n})^{*} \sum_{\mathrm{K}=0 \text { toM-1 }}\left(\mathrm{w}(\mathrm{K})^{*}\left(\mathrm{e}^{-\mathrm{j} \mathrm{k} \Omega}\right)\right) \ldots \ldots \ldots \ldots \ldots \text {...... [6 [ }
$$

Where $s(n)=$ source signal

This algorithm is also known as Capon's method which was suggested to obtain better resolution compared to delay- sum method [8].

\section{ii. Music Algorithm (Multiple Signal Classification)}

The principle behind this algorithm is to separate the Eigen of the co-variance matrix of the sensor outputs with the help of co-variance of noise. The sensor output correlation matrix is divided into signal-noise sub spaces and noise sub spaces. This is a high resolution subspace algorithm which is used for estimating the arriving signals and their direction [8] [9]. The orthogonality of Eigen values for Hermitian matrices results in zero noise as they are orthogonal to the signal direction. The number of signals that can be detected is restricted by the number of elements in the microphone array i.e. $\mathrm{N}$ element microphone array can detect up to $\mathrm{N}-1$ uncorrelated signals [5].

\section{iii. Root Music Algorithm}

It is a polynomial variation to Music Algorithm [9] which has an advantage over the same for linear arrays. It also includes analysis of the covariance matrix. This algorithm evaluates the roots of the spectrum such that the roots which are closer to the unit circle are assigned to the desired signal while the remaining roots further from the unit circle are assigned to noise. The angles of the signals are then computed with the help of these roots [5] so as to beam form the signals.

iv. ESPRIT Algorithm (Estimation of Signal Parameter via Rotational Invariance Technique)

ESPRIT explores the rotational invariance, created by two sub-arrays, in the signal sub-space [9]. It uses two arrays in the sense that the second element of each pair is displaced by same distance with respect to the first element. One array can also be used to realize this algorithm by selecting subset of the elements. Here two arrays are deduced from the received signals. The correlation matrices of these two arrays are formed which are used to compute the Eigen values. These Eigen values are then used for calculating the angles of the signals [5] so as to beam form the signals.

\section{v. LMS Algorithm}

This algorithm uses a gradient based method on the principle of updating the filter coefficients by calculating the error in the signals. In some applications, adaptive coefficients are required as some parameters in the processing operation will be unknown. This process is basically a criterion for performance. These filters are important as they now routinely find application in mobile and other communication devices. The main idea behind an LMS adaptive algorithm is that a variable filter extracts an estimate of the desired signal [10]. The following assumptions are made with respect to LMS algorithm.

a. The input signal is a combination of desired signal, $d$ (n) and interference, I (n). 
b. The variable filter whose impulse response is equal to the filter coefficients has a FIR structure.

c. Error signal is the difference between desired and estimated signal.

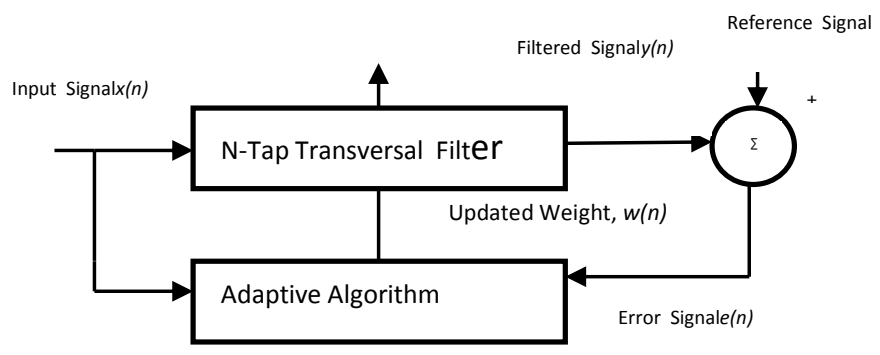

Fig.2 Adaptive Filter [10]

Fig.2 shows an adaptive filter which is used to estimate desired signal from the input signal and impulse response. The coefficients of this adaptive filter adjust and are updated with every input sample. Many algorithms are proposed, where Least Mean Square (LMS) algorithm is the most simpler and popular method.

The basic fundamental of this algorithm is explained by the equations below.

$$
\begin{aligned}
& y(n)=\sum c(k) * x(n-k) \ldots \ldots \ldots \ldots . . . E q .7[11] \\
& e(n)=d(n)-y(n) \quad \ldots \ldots \ldots \ldots . . E q .8[11] \\
& c(n+1)=c(n)+\mu x(n) * e(n) \ldots . . E q .9[11]
\end{aligned}
$$

Eq.7 gives the filtered output while the error signal is obtained according to the Eq.8. Eq.9 is used by the adapting algorithm to calculate the updated coefficients [12] [13]. LMS algorithm is preferred for its simplicity and the convergence of filter depends on the step size, $\mu$. The weight vector seem to converge and stay stable for

$0<\mu<1 / \lambda_{\max }$

Where $\lambda_{\max }=$ largest Eigen value of correlation matrix $R$

If $\mu$ is small, it takes longer time to converge while for larger $\mu$, the algorithm may lead to faster convergence but lesser stability [13]. So the performance of filter depends on the step size.

\section{DISCUSSION}

In order to obtain good SNR, where direction of signal is not of priority, one would prefer the much simpler and efficient LMS algorithm than the other complex algorithms. The subspace algorithms like MVDR, MUSIC, Root MUSIC and ESPRIT are complex as it takes into account the direction of signals. These can be analyzed by using various parameters like number of snapshots, SNR, number of antenna array elements etc [9]. Review of relevant articles, has shown that MUSIC algorithm has better performance than MVDR and Root-MUSIC even though ESPRIT is more robust with respect to array perfection. ESPRIT also has less complexity and storage requirement than MUSIC. MUSIC algorithm tends to show less sensitivity to angular separation as compared to Root MUSIC and MVDR. Both MUSIC and ESPRIT provide high resolution, more accuracy and is not limited to physical size of array aperture [9].

Microphone array processing techniques has the potential to relieve users from the need of having close-talking microphones [14]. These array sensors combines signals either constructively or destructively and functions as spatial filter using the above algorithms to suppress interference [15].

\section{CONCLUSION}

The literature study on acoustic beam forming gives an idea about the different algorithms employed. These can be used to produce high performance signals which have good quality SNR for long distance communications. Theoretical comparison of these different algorithms is also attempted in this paper. This would enable the use of appropriate algorithms for different applications. Further, these analyses provide the theoretical base for implementation of beam formers in FPGA. Along with the comparative results of all these algorithms, it also enables us to study these methods in a two stage model. Two stage designs can further be compared with single stage designs for their directivity and noise removal.

\section{REFERENCES}

[1] Emanuel A.P. Habets, Jacob Benesty, "A Two Stage Beamforming Approach for Noise reduction and Dereverberation," IEEE Transactions on Audio, Speech, and Language Processing, Vol. 21, N0. 5, May 2013, pp. 945-958.

[2] Michael L. Seltzer, Richard M. Stern, "Subbands Likelihood- Maximizing Beamforming for Speech Recognition in Reverberant Environments," IEEE Transactions on Audio, Speech and Language Processing, Vol.14, No.6 Nov 2006, pp. 2109-2121.

[3] Alaa Abdeen, Laura Ray, "Design and Performance of a real-time Acoustic Beamforming System,” IEEE, 2013.

[4] Jacob Benesty, Jingdong Chen,etal, "On MicrophoneArray Beamforming From a MIMO Acoustic Signal Processing Perspective," IEEE Transactions on Audio, Speech, and Language processing, Vol.15, No.3, Mar 2007, pp.1053-1065.

[5] Tshilidzi Mukwevho, Jaco Jordaan, Guillaume Noel, "Advanced Beamforming Techniques for Acoustic Source Localization," IEEE AFRICON, 23-25 September 2009, pp.1-6.

[6] T.S.Jeyali Laseetha,Dr. R.Sukanesh, "A Novel Robust Adaptive Beamformer using Diagonal Loading", International Journal of Computer Applications, Volume 19- No.5, April 2011,pp.35-40.

[7] Balasem. S.S , S.K.Tiong, S. P. Koh, "Beamforming Algorithms Technique by Using MVDR and LCMV," World Applied Programming, Vol (2), Issue (5), May 2012, pp.315-324

[8] S. N. Bhuiya, F. Islam, and M. A. Matin, "Analysis of Direction of Arrival Techniques Using Uniform Linear Array," International Journal of Computer Theory and Engineering, Vol. 4, No. 6, December 2012, pp. 931-934.

[9] Tanuja S. Dhope (Shendkar), Dina Simunic, Radovan Zentner, "Comparison of DoA Estimation Algorithms in SDMA System,” AUTOMATIKA, Vol 54, No.2, 2013, pp.199-209.

[10] S.S.Godbole, P.M. Palsodkar, V.P.Raut, "FPGA Implementation of Adaptive LMS Filter," Proceedings of SPIT-IEEE Colloquium and International Conference, pp. 2226-2229.

[11] Jashvir Chhikara, Jagbir Singh, "Noise cancellation using adaptive algorithms," International Journal of 
Modern Engineering Research, Vol.2, Issue 3, May-June 2012, pp. 792-795.

[12] Sayed A. Hadei, M. Lotfizad, "A Family of Adaptive Filter Algorithms in Noise Cancellation for Speech Enhancement," International Journal of Computer and Electrical Engineering, Vol.2, No.2, April 2010

[13] Asit Kumar Subudhi, Biswajit Mishra, Mihir Narayan Mohanty, "VLSI Design and Implementation for Adaptive Filter using LMS Algorithm," International
Journal of Computer and Communication Technology, Volume-2, Issue-VI, 2011,pp. 84-88.

[14] Kenichi Kumatani, John McDonough, etal, "Beamforming with a Maximum Negentropy Criterion," IEEE Transactions on Audio, Speech, and Language processing, Vol.17, No.5, July 2009, pp.99.

[15] Kenichi Kumatani, John McDonough, Bhiksha Raj, "Microphone Array Processing for Distant Speech Recognition," IEEE signal Processing Magazine,November 2012,pp.127-140. 